\title{
Long-term safety and efficacy of Canakinumab in cryopyrin-associated periodic syndrome (CAPS) patients: results from beta-confident registry
}

\author{
J Kuemmerle-Deschner ${ }^{1 *}$, H Hoffman²${ }^{2}$ PN Hawkins ${ }^{3}$, T van der Poll $^{4}$, UA Walker ${ }^{5}$, A Speziale ${ }^{6}$, HH Tilson ${ }^{7}$ \\ From 8th International Congress of Familial Mediterranean Fever and Systemic Autoinflammatory Diseases \\ Dresden, Germany. 30 September - 3 October 2015
}

\section{Background}

CAPS encompasses a spectrum of three phenotypes: familial cold auto-inflammatory syndrome (FCAS), Muckle-Wells syndrome (MWS), and chronic infantile neurologic cutaneous and articular syndrome/neonatal onset multisystem inflammatory disease (CINCA/ NOMID)[1]. The $\beta$-Confident Registry, the largest CAPS cohort documented in a registry, enrolled the last patient in December 2014. Here, we report interim data for the complete cohort of enrolled patients.

\section{Objectives}

To monitor the overall safety of canakinumab (CAN) focusing on SAEs including serious infections, vertigo, malignancies, and hypersensitivity reactions.

\section{Patients and methods}

The registry protocol does not mandate any visits or procedures, but records all observed and reported AEs and SAEs or AEs potentially CAN-related. Cumulative safety data are reported as incidence rate per 100 patient-years (IR/100 pyr). Data is partial for 11 patients due to the cut-off date for the analysis and will be updated at a later date. Efficacy was measured using physician global assessments (PGA).

\section{Results}

288 patients were enrolled with a mean \pm SD duration of $193 \pm 72$ weeks. Of these, 21 (7.3\%) patients discontinued CAN: 5 each due to AE, poor efficacy and patient preference; and 6 due to unknown reasons. The IR/100 pyr for overall AEs was 100.0. FCAS patients had the lowest AE

${ }^{1}$ University Hospital Tuebingen, Tuebingen, Germany

Full list of author information is available at the end of the article
IR/100 pyr (60.9) compared with MWS (IR/100 pyr 107.2) and NOMID (IR/100 pyr 120.3) patients. The most common types of AEs were infections and infestations (IR/100 pyr 36.7). Vertigo was reported by 19 patients (IR/100 pyr 3.7). 117 SAEs were reported by 62 patients (IR/100 pyr 15.0), with infection being the most common (IR/100 pyr 4.1). One death (metastatic rectal adenocarcinoma in $76 \mathrm{yr}$ old MWS patient) was reported. Of 18 patients receiving pneumococcal vaccinations (PPV), 13 (72\%) reported a local post-PPV injection site reaction, of which 5 were considered as serious. Based on PGA, nearly half the patients had no disease activity while most others had mild/moderate disease activity. Similarly, disease activity was mostly absent in NLRP3 mutation negative CAPS patients $(n=14)$ treated with CAN. There was no evidence of loss of effect with time. Further analyses of this cohort are ongoing.

\section{Conclusions}

Canakinumab demonstrated a safety profile consistent with that observed in the clinical trial program and provided continued effectiveness in CAPS patients for up to 5 years. Canakinumab therapy was also effective in NLRP3 mutation negative CAPS patients.

\footnotetext{
Authors' details

'University Hospital Tuebingen, Tuebingen, Germany. ${ }^{2}$ University of California,

La Jolla, CA, USA. ${ }^{3}$ University College London Medical School, London, UK.

${ }^{4}$ University of Amsterdam, Academic Medical Center, Amsterdam, Netherlands.

${ }^{5}$ University Hospital, Basel, Switzerland. ${ }^{6}$ Novartis Pharma AG, Basel, Switzerland.

${ }^{7}$ University of North Carolina, Chapel Hill, USA.
}

Published: 28 September 2015

\section{Reference}

1. Kuemmerle-Deschner JB, et al: Arthritis Res Ther 2011, 13(1):R34. 
Submit your next manuscript to BioMed Central and take full advantage of:

- Convenient online submission

- Thorough peer review

- No space constraints or color figure charges

- Immediate publication on acceptance

- Inclusion in PubMed, CAS, Scopus and Google Scholar

- Research which is freely available for redistribution

Submit your manuscript at www.biomedcentral.com/submit
() Biomed Central 